\title{
Biomarkers of Major Depressive Disorder: Knowing is Half the Battle
}

\author{
Sahil Malik', Ravinder Singh', Govind Arora', Akriti Dangol', Sanjay Goyal ${ }^{2}$ \\ ${ }^{1}$ Chitkara College of Pharmacy, Chitkara University, Rajpura, Punjab, ${ }^{2}$ Department of Internal Medicine, Government Medical College, Patiala, \\ India
}

\begin{abstract}
Major depressive disorder (MDD) is a heterogeneous disease which is why there are currently no specific methods to accurately test the severity, endophenotype or therapy response. This lack of progress is partly attributed to the complexity and variability of depression, in association with analytical variability of clinical literature and the wide number of theoretically complex biomarkers. The literature accessible, indicates that markers involved in inflammatory, neurotrophic and metabolic processes and components of neurotransmitters and neuroendocrine systems are rather strong indicators to be considered clinically and can be measured through genetic and epigenetic, transcriptomic and proteomic, metabolomics and neuroimaging assessments. Promising biologic systems/markers found were i.e., growth biomarkers, endocrine markers, oxidant stress markers, proteomic and chronic inflammatory markers, are discussed in this review. Several lines of evidence suggest that a portion of MDD is a dopamine agonist-responsive subtype. This review analyzes concise reports on the pathophysiological biomarkers of MDD and therapeutic reactions via peripheral developmental factors, inflammative cytokines, endocrine factors and metabolic markers. Various literatures also support that endocrine and metabolism changes are associated with MDD. Accumulating evidence suggests that at least a portion of MDD patients show characteristics pathological changes regarding different clinical pathological biomarkers. By this review we sum up all the different biomarkers playing an important role in the detection or treatment of the different patients suffering from MDD. The review also gives an overview of different biomarker's playing a potential role in modulating effect of MDD.
\end{abstract}

KEY WORDS: Major depressive disorder; Brain-derived neurotrophic factor; Insulin-like growth factor I; IL-1ß; Mood disorders.

\section{INTRODUCTION}

Beginning around 1990, drug development scientists were progressively curious about quantitative markers of malady activity, maybe influenced by the requirement to get speedy quantitative indications of drug action to hurry the event of recent anti-depressive agents [1].

The terminology "biomarkers" have been used since 1970s. In that case, the word was used to show existence of biological origin material. The first clinical use of biomarkers was done in the year 1977 in an article named,

Received: April 14, 2020 / Revised: July 2, 2020

Accepted: September 15, 2020

Address for correspondence: Ravinder Singh

Chitkara College of Pharmacy, Chitkara University, Chandigarh-

Patiala National Highway (NH-64), Punjab 140401, India

E-mail: ravi.jaura@gmail.com

ORCID: https://orcid.org/0000-0003-1565-9740
"Tumor biomarkers of value in the management of gynaecologic malignancy will also be correlated with clinical course" $[2,3]$. Throughout all these years, brain diseases have conquered the age of explosive development. APHD: Applied Psychology and Human development at OISE (Ontario Institute for Studies in Education) have stated that neurodegenerative disorders are the most prevalent illnesses impacting public health $[4,5]$.

The aetiology of depression is, as is the case with any other psychiatric disorders, incredibly complex, involving psycho-social, genetic, epigenetics and neuroendocracy $[6,7]$. This complexity has direct consequences for our accuracy in the diagnosis and subtypes of depression [8], for our comprehension of pathophysiology [9] and for our ability to design and choose effective treatment strategies [10]. The pharmacological treatments currently available are primarily intended for improved neurotransmission

(c) This is an Open-Access article distributed under the terms of the Creative Commons Attribution Non-Commercial License (http://creativecommons.org/licenses/by-nc/4.0) which permits unrestricted non-commercial use, distribution, and reproduction in any medium, provided the original work is properly cited. 
dependent on monoamine, but as shown in the STAR-D report, patients that have a greater burden of disease needed. Pharmacotherapy, which does not always result in remission [11]. In fact, major side-effect profiles [12] and delayed initiation of operations [13] impact conformity with the regulations are possible. Late on the production of new, non-monoamine-based depression approaches, including the melatonergic mechanism [14] or glutamate method $[15,16]$, has become increasingly essential. Other interventions, including psychotherapy [17], electroconvulsive therapy [18], magnetic transcription, stimulating the vagus nervous and stimulation of the deep brain [19], are not clear but are all effective to different degrees.

\section{BIOMARKERS IN DEPRESSION: AN INSIGHT}

To gain a full understanding of the molecular pathway and its contribution to psychiatric disorders, multiple biological "levels" in an approach popularly referred to as the approach to "omics" are considered to be important to assess [20]. Nevertheless, while each system can be inspected at every omics level, the optimal measuring sour- ces vary significantly at every level. For example neuroimaging, offers a forum to evaluate the brain structure or activity implicitly while blood protein tests explicitly measure markers. Transcriptomic [21] and Metabolomics [22] are increasing in importance, with possible numbers of markers being measured and the Human Microbiome Project also aims to classify both genetic compositions and microorganisms in humans [23]. New technologies increase our ability to measure this, even though additional sources, such as hormones such as cortisol (providing a chronic indication), cerebrospinal fluid, urine and saliva, [24-26] as well as blood. Hormones such as cortisol are now being tested in the hair or fingernails [27] as well.

Insight of some biomarkers is well summarized in the Table 1.

\section{ADVANTAGES OF BIOMARKERS}

The advantages that biomarkers possess include its simplicity and less expensiveness for analyzing final clinical endpoints which can be measured on a repeated basis and within lesser time period $[28,29]$. The creation of biomarker panels to recognize different peripheral/serum growth factors, cytokines, hormonal and metabolic mark-

Table 1. Overview of recent insights into biomarkers for depression

\begin{tabular}{|c|c|c|c|}
\hline $\begin{array}{c}\text { Serial } \\
\text { number }\end{array}$ & $\begin{array}{c}\text { Biomarker } \\
\text { system }\end{array}$ & Studies on biomarker & Reference \\
\hline 1 & Inflammation & $\begin{array}{l}\text { - Proinflammatory markers are higher in depression than controls. } \\
\text { - Inflammation tends to decrease with antidepressant treatment. } \\
\text { - Inflammation seems more aberrant in treatment nonresponders. } \\
\text { - Anti-inflammatory treatments reduce depression severity. }\end{array}$ & {$[31],[34],[81],[119]$} \\
\hline 2 & Neuroendocrine & $\begin{array}{l}\text { - HPA axis appears overactive in people with depression. } \\
\text { - Atypical depression may show hypocortisolism. } \\
\text { - High cortisol may predict a poorer response to psychological therapy and } \\
\text { pharmacologic therapy. }\end{array}$ & {$[26],[35],[66]$} \\
\hline 3 & GF & $\begin{array}{l}\text { Some neurotrophic factors are reduced in depression compared to controls (BDNF, } \\
\text { NGF, and GDNF). } \\
\text { - Some GFs may be overproduced in depression (VEGF, bFGF). } \\
\text { - Neurotrophic factors appear to increase alongside treatment, regardless of response. }\end{array}$ & {$[26],[32],[66]$} \\
\hline 4 & Neurotransmitter & $\begin{array}{l}\text { - There is widespread increased } 5-\mathrm{HT}_{1 \mathrm{~A}} \text { binding in people with depression that can be } \\
\text { influenced by treatment. } \\
\text { - Monoamines interact to influence cognitive function and responses to stress; may } \\
\text { provide mechanisms of TRD. }\end{array}$ & {$[27],[28],[96]$} \\
\hline 5 & Metabolic & $\begin{array}{l}\text { - Depression is associated with altered metabolic profiles. } \\
\text { the promise of metabolic m,arkers for improving depression treatments is limited by } \\
\text { - Atypical depression linked with greater metabolic abnormalities. }\end{array}$ & {$[18],[96],[97]$} \\
\hline
\end{tabular}

GF, growth factor; BDNF, brain-derived neurotrophic factor; NGF, nerve growth factor; GDNF, Glial cell-derived neurotrophic factor; VEGF, vascular endothelial growth factor; bFGF, basic fibroblast growth factor; TRD, treatment-resistant depression; BMI, body mass index. 
ers and to protect several biological anomalies leading to major depressive disorder (MDD)'s variability and therapeutic responses may therefore be a promising option to a single biomarker strategy. Some of the important biomarker are discussed in this review which are mainly growth factors, cytokines, metabolites and metabolic marker which could prove to be a very potent one [30]. Clinical studies have proven that biomarkers have contrasted improvement in patients with MDD vs. Control [31,32]. Therefore, the absence of straightforward associations between MDD and other disorders of comorbid depression is a significant drawback in biomarker collection for depression. In order to determine a peripheral signature for MDD, the identification of the latest biological MDD target markers would enable the accurate identification of cytokines, growth factors, hormones, and other protein markers in plasma samples. Biomarker platforms will then enable the detection of a peripheral characteristic that separates MDD sub-types, MDD from other diseases, growth regulators, hormones and other protein indicators for the same predictions. The monitoring of several factors provides a more thorough evaluation, thereby identifying a range of factors which better characterize disease conditions and specific symptoms of disease. This detail may also be used to directly handle the growth factor or b cytokine levels changed or neutralized. In basic terms, while it is not possible that single biomarkers adequately distinguish between distressed and non-depressed participants, panels with many biomarkers are much easier.

Several pharmacogenomics experiments have examined the moderating impact of particular genetic variance on antidepressant medication response. Sequenced Treatment Alternatives to Relieve Depression (STAR*D), STAR*D study reported by [32], reported the response or outcome of SSRI citalopram, subsequent medicinal therapies, or combinations of therapy. STAR*D explores polymorphisms in common nucleotides. They contained the protein binding FK506-5 (FKBP5), the ionotropic kainate-1 glutamate receptor (GRIK1) and 4 (GRIK4), aspartate n-methyl receptor-2A (GRIN2A), Genus-K potassium channel member-2 (KCNC2) (six SNPs) and long/quick vector transporters (SLC6A4) with the five-hydroxytriptamine receptor-2A (HTR2A). Some proteins, including cyclic-AMP-reaction-binding protein-1 (CREB1), ionotropic glutamate-receptor-AMPA-3 (GRIA3) and GRIK2, were also linked with treatment-emergent suicidality [33] .
Biomarkers are persistent and suggest disease before, after, and also after recovery of the condition begins, these are helpful in determining whether would establish a disease. State biomarkers are acute, indicate a person's health condition and are active before and after the disease but are reduced in remission. Biomarkers of the Endophenotype are a subtype of feature bio-markers, based on ties between genes and other suicidal phenotypes [34]. A realistic solution to mental condition research (e.g., depression) by incorporating multiple layers of quantitative biologic and psychological contextual data into a framework is the Research Domain Criteria (RDoC) program of the National Institute of Health [35].

\section{Accuracy of Biomarker Measuring}

Good predictive value is the probability that a person with the good biomarker outcome may have depression and a negative predictive value in turn is likely to have no depression [36]. The potential will have predictive levels as biomarkers are correlated with the patient's likelihood of depression. A further caution is that the biomarker is neither present nor inaccessible when psychiatric problems, such as dependency are involved; instead, it is found beyond the baseline levels or normal comparison level obtained from the control data which were not exposed to the disease. Therefore, while in psychology the idea of recovery is applicable, it is somewhat specific than other areas of medicine. In these situations, latent class analysis is an approach that is more feasible, integrating numerous flawed biomarker analytics [37]. This will in fact take the form of a biomarkers panel already established and tested by study groups for accuracy [38].

\section{METHODOLOGY FOR THE REVIEW}

With the keywords such as "Major depressive disorder; antidepressant; brain-derived neurotrophic factor (BDNF); insulin-like growth factor I (IGF-1); interleukin $1 \beta$ (IL-1 $\beta$ ); serum" a systematic literature analysis was carried out of the corpus of the PubMed and EMBASE (Elsevier) up until the year 2020. The review was carried out using the above keywords to gather the latest articles to understand the urgency of the matter. Based on MDD data, the searches have been limited to those reported in previous 10 years, which were verified earlier. Because of the vast number of documents contained in the grey literature, Google Scholar's 
scope was confined to titles; therefore, no additional limit was applied in the PubMed or Embase search. Extracting details from the archive files using the endnote, title screening, and abstract screening was performed using the Rayyan QCRI web software Systematic Reviews. The entire text was then checked for future use in the systematic examination. Reports evaluated for concerning to the biomarkers which can be potentially used for MDD.

\section{Biomarkers for Depressive Disorders}

\section{Proteomic markers}

Proteins are the cell's big actors modulating some major receptors like 5-HTT, dopaminergic; NMDA, GABA and enzymes linked to oxidative stress are examined as proteomes in depression. Previous findings in post-mortem and in vivo imaging have found 5-HTT expression reduced in different regions of the brain in terms of proteomic function [39] and furthermore, psychotherapy induced an increase in 5-HTT expression more so in patients with high baseline severity scores compared to patients with low scores but this did not correlate with symptomatic improvements $[40,41]$. As we note, although the impact of antidepressants on 5-HTT expression has been preclinical investigated [42], no clinical trials have been carried out. One of the fascinating features of serotonergic receptors and transporters is that they rely on the clustering of so-called lipid rafts [43], and the size and amount in lymphocytes in patients has dramatically increased in contrast with stable controls [44]. A variety of experiments have been carried out in peripheral circulation with activation of dopamine receptors D3, D4 and D5 [45]. However, only the D4receptor has been seen to date in the sense of depression. The function of the D4 receptor in depression pathophysiology remains well known but data has been shown that certain polymorphisms are involved [46].

A heterogeneous framework comprising of many sub-units [47] is the NMDA receiver. Increasing mRNA expression in subunits NR1, NR2A, N2C, N2D and N3A has been documented by a recent transcriptomic analysis on post-mortem research samples in female depression patients dorsally-lateral prefrontal cortex [48]. It is noteworthy that in patients with depression, the frequency of mRNA in NR2B was higher. At the other side, proteomic analyzes have reported decreased rates in the NR1, NR2A and NR2B subunits in the medial temporal lobe and the prefrontal cortex, even in post-mortem samples [49]. The rates of Plasma GABA to be depression biomarkers have been reported [50], although there is evidence that they may fluctuate in reaction to SSRI [51] therapy. In addition, higher GABA plasma baseline rates were found to be predictive of ECT response [52]. The GABA synthesis enzyme in patients with depression is equivocal in postmortem evidence of glutamic acid decarboxylase production in [53] patients [54]. Due to the opposing position of glutamate and GABA, and the probability of signaling between neurotransmitters [55], a glutamate/GABA imbalance ratio may also be a depression function. In either case, this hypothesis is backed by contradictory data stemming largely from trials utilizing magnetic resonance spectroscopy [56], which can be a long and expensive method, rendering it a point of treatment strategy unsuccessful [57].

Growth factors: Several clinical studies for gene expression altering as well as peripheral levels have shown several growth factors in patients with major depressive disorder, while medications are usually ineffective. Factors of production include the insulin-like growth factor, BDNF [58]. The most often studied MDD growth factor is BDNF. Synaptic plasticity and movement control by BDNF [59]. It is liberated from nerves and can be transferred by peripheral cells, such as leukocytes, cells and platelets $[60,61]$. Sufficient evidence indicate that BDNF is essential in stress response and has protective effects from brain stimulus changes. Animal experiments have also shown a significant decrease of hippocampal BDNF expression owing to physical or behavioral tension. Chronic stress presence decreases neurogenesis and resistance by causing down-regulation of animal experiments in the BDNF pathways $[62,63]$. Combined, a reduction in hippocampal BDNF activity is suspected of being specifically linked to stress disturbance and MDD pathophysiology. A variety of experiments and meta-analysis indicate that BDNF levels in serum and plasma are higher in depressed populations. Higher corticosteroid concentrations are likely to contribute to decrease BDNF levels because GRs are negatively affected by the BDNF gene [64]. MDD and the bipolar disorder are known to have same behavior pattern, this is supported through the serum BDNF levels were recorded to be lower than in MDD during the bipolar depression [65]. 
Research and meta-analyses regularly indicate that antidepressants and ECT reverses the drop in the BDNF level which occurs in depression. In addition, initial BDNF levels have been found to be higher in patients with MDD who respond to treatment. A meta-analysis found that BDNF levels could be used as a determinant for successful treatment with antidepressants, as serum BDNF levels remained unchanged for patients who did not react to antidepressants, but who increased in therapy responders and in patients who achieved recovery [66].

BDNF, the Vascular Endothelial Growth Factor, the fibroblast growth factor (FGF) and the VGF interact in neuronal development as well as in differentiation, repair and synaptic plasticity. None of these are implicated in depression, both of them are impaired by drugs and are special to the role as biomarkers for their presence in brain and peripheral circulation [67-70]. In the dorsolateral prefrontal cortex, in the anterior cingulate cortex, the hippocampal cortus and locus coeruleus, MD-patients were documented dysregulating the transmission of multiple FGF-transcripts (FGF 1, FGFR 1, FGF2, FGFR2, FGFR3) relative to the controls on safe medicine [71-74].

The peripheral growth factor and the cytokines are seen to have major important consequences in the animal models and control behavioral responses. IGF-1 secretion is produced predominantly in the liver and successfully transmitted via the endocytic drug receptor to the brain. Peripheral IGF-1 enhances development neurogenesis and antidepressants. A variety of secondary tissues such as coronary, kidney, heart, liver, and brain tend to describe the BDNF amount in bloodin Figure 1.

\section{Cytokines and inflammatory markers}

Inflammatory pathways are widely researched during stress-related illnesses and depression. In MDD pathophysiology, inflammation is seen to play a significant role. Many factors that considered important for MDD's etiopathogenesis are influenced by inflammatory mediators. This means that inflammatory markers can be used to diagnose and treat depression $[75,76]$. Cytokines, in addition to being crucial in the development of brain, play a major part in the upkeep of natural functioning of brain by encouraging neurogenesis, neuronal integrity, as well as synaptic remodeling. The effect of neurotransmitters often causes behavioral responses. Peripheral immune cells and adipose tissue cytokines have already been reported to enter the central nervous system as a significant medical mode of intervention in certain parts of the Blood Brain Barrier (BBB). The brain produces mainly microglia and astrocyte cytokines [77].

It is known to be that the inflammatory variables might also initiate depression through following mechanisms:

- Altering neurotransmitters,

- Diminishing serotonin as well as activating glutamate toxicity,

- Abolishing neurogenesis by lowering down BDNF activity, or

- Expanding HPA axis functioning.

The regularly and consistently increased in case of depression is IL-6 and C-reactive protein [78-80]. Higher standards just or no gaps in European research [81]. Nonetheless, a recent study has suggested unique translation results for IL-1 $\beta$ backed by a highly important trend of elevated IL-1 $\beta$ ribonucleic acid prediction of weak antidepressant reaction [82]. Among depressive subjects, chemical monocyte-chemo attractor protein-1 raised rates in a meta-analysis. IL-8 has been reported as elevated in those with severe depression [83]. Similar rates of improvement have arisen between early and non-responding patients in IL-10 and interferon gamma in treatment [84] and IL-4 and IL-2 have been lowered in conjunction with symptom remission [85]. For IL-6, IL-1 $\beta$, $\mathrm{IL}-10$ and C-reactive protein, the meta-analysis revealed slight decreases along with therapy [86,87]. In fact, tumour necrosis factor- $\alpha$ can be decreased even with responder therapy, so an index of composite markers can suggest elevated praks of inflammation in patients that do not response to therapy later on. About the majority of the work investigating inflammatory markers and clinical reactions requires pharmacological clinical tests, however [88-90].

\section{Metabolic and Oxidative stress markers}

Leptin, adiponectin, ghrelin, triglycerides, lipoprotein (HDL), creatine, insulin and albumin [91] are the main biomarkers linked with this metabolic condition. Some of those correlations have been tested for depression: leptin [92] and ghrelin [93] are shown to be smaller in depression than in peripheral controls and may be improved with antidepressants and recovery. The resistance to insulin may be improved in depression, albeit lower [94]. Lipid levels, including HDL cholesterol, tend to have 


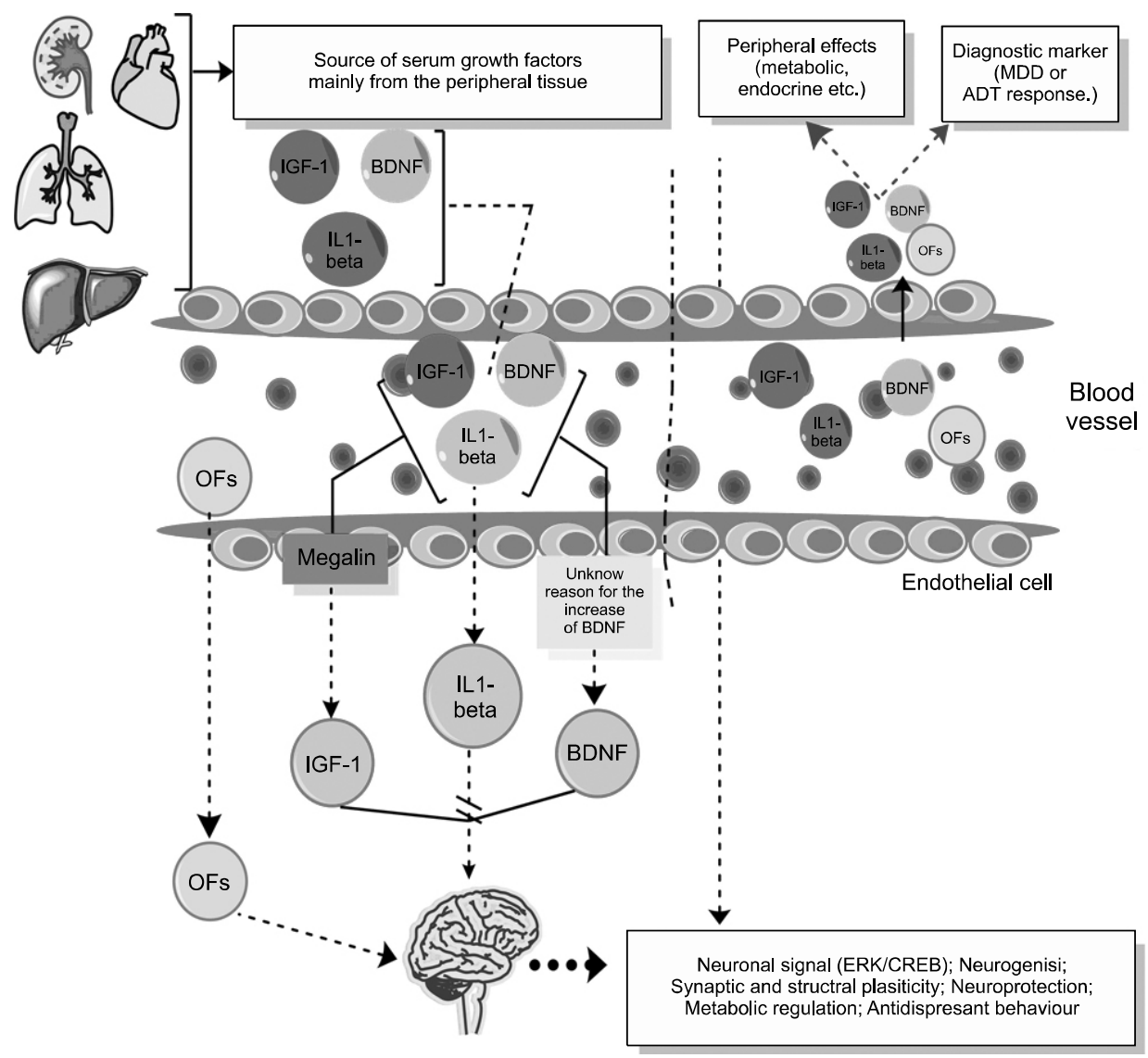

Fig. 1. Peripheral growth factors and pro-inflammatory cytokines exert opposing influences on antidepressant-like cellular (i.e., neurogenesis) and behavioral responses in case of MDD. Sustainable IGF-1 is primarily produced in the liver and transmitted by endocytic megalin in the brain effectively. Peripheral IGF-1 increases neurogenesis in the adult hippocampus and produces behavioral antidepressant responses. Several secondary tissues, including the skin, kidneys, lung, liver, and cortex, lead to blood BDNF rates. Administration of peripheral BDNF enhances adult neurogenesis and induces physiological responses comparable to the antidepressant. Consequently, it can be determined whether the results are inducted through direct or indirect pathways (i.e., blood BDNF to the brain). In contrast, stress exposure leads to inflammatory processes, including increased macrophage cytokine release. Circulating cytokines, such as IL-1b, decrease the neurogenesis of an adult hippocampus and lead to depressive conduct. There is also an opposite effect on developmental (i.e., neurogenesis) and compartmental reactions on peripheral growth factors and proinflammatory cytokine.

ADT, antidepressant; BDNF, brain-derived neurotrophic factor; IGF-1, insulin-like growth factor-1; IL-1 $\beta$, interleukin $1 \beta$; MDD, major depressive disorder; OFs, other factors; ERK, extracellular-signal-regulated kinase; CREB, CAMP response element binding protein.

shifted in certain people with depression, particularly those without a comfortable physical condition [95]. There have been also reported hyperglycemia [96] and hypoalbuminemia depression. Investigation of general metabolic diseases with limited molecular metabolomics panels in order to identify a strong biochemical signature in clinical disorders is becoming increasingly popular. A sequence of metabolites that display enhanced glucose-lipid signals were highly predictive of an MDD diagnosis [97] in a recent analysis using artificial intelligence models [98], which backed previous studies [99].

It is proposed that oxidative stress and antioxidant de- fenses are improved in MDD and those particular oxidative stress components play an active function in the pathophysiology of depression [100].

Recent studies have shown that there is an increased level of malondialdehyde in patients having frequent depression than in those who have faced depression just once. The second criterion which is studied as an oxidative stress marker in the patients suffering with MDD is the activity of Superoxide dismutase (SOD). Hence, it can be concluded that serum SOD is reduced or erythrocyte SOD is elevated in patients suffering from depression [101]. 
A variety of cross-sectional experiments have been found to be related to 8-hydroxy-2-deoxyguanosine (8-OHdG). Urinal rates [102] and serum were higher among those who experienced severe depression, relative to the stable control groups [103] of 8-OHdG. 8-OHdG levels also have a positive correlation with severity of depression [104], and those with recurrent episodes of depression were more likely that those with single episodes [105]. The levels of OHdG also have a positive correlation to the severity of depression. The $8-\mathrm{OHdG}$ rises may be a clinically recognized characteristic of depression, since there were no variations in group groups with depressive symptoms [106,107].

Levels of isoprostanes are elevated in patients suffering from depression as demonstrated by higher urinary concentrations of 8-iso-PGF $2 \alpha$ It has been studied in an analysis that the impact of antidepressant treatment on the isoprostane rates. Following 8 weeks of therapy for bupropion or sertraline in patients with severe depression the excretion of F2 isoprostane improved dramatically. F2 increases were also found to be associated with enhancements in depression severity [108].

\section{Endocrine markers}

Energy balance hormones: Studies on levels of hormones, such as leptin and ghrelin which regulate the balance of body energy in MDD have been performed progressively in recent decades Hormones including leptin and ghrelin distribute intelligence around homeostatic peripheral energy levels to the brain, Serum leptin levels reducing chronic stress have been recorded. In fact, the animal therapy of acute leptin has also been shown to yield therapeutic results followed by decreased hippocampal production of BDNF. Leptin rates in MDD patients are not obvious. The amounts of may be mainly studied through blood components. Leptin have been decreased, raised or remains unchanged. Ghrelin's depression results was often inconsistent. Studies have found that ghrelin levels have reduced or enhanced and others have investigated that it improves or reduces with antidepressant therapy. Therefore, leptin and ghrelin-related observations are still insufficient to enable them to represent as biomarkers in depressed patients [109].

\section{Structural and Functional Imaging Findings}

In particular, in the brain structures linked to emotion and mood regulation, structural and functional changes in MDD have been identified. There is no general loss in brain volume of people with MDD, of contrast with bipolar disorder. MDD patients have recorded morphological anomalies, including lower volume in several brain structures including hippocampus, baseline ganglia, and precinct cingulate anterior cortex, reduced cortical thickness, reduced volume of gray matter, and decreased integrity of white matter [110].

Cortisol is the most widely researched HPA axis biomarker specifically for depression. Numerous studies focused on the different HPA evaluations; broadly speaking, these indicate that hypercortisolemia is linked with stress and a recurrent attenuation of cortisol awakening responses $[111,112]$. It is confirmed by a new study of persistent hair cortisol rates, which confirms cortisol hyper activeness theory in depression but hypo activity in certain disorders such as panic disorder. In comparison, higher cortisol rates can indicate fewer psychological reaction [108] and more antidepressant treatment $[113,110]$ in fact. Historically, dexamethasone inhibition studies, since cortisol non-suppression was correlated with a lower risk of eventual relapse, have become the first choice neuroendocrine tagging of prospective therapy progress. More possible candidates of biomarkers representing biological processes are precisely tested using neuroimagery or genetics. Since genomic discrepancies between distressed and non-depressed populations are not so large and significant [114], it may be more valuable to take modern genetic strategies such as polygenic scores [115] and telomere length [116]. Circadian processes or Chrono biological biomarkers from different methods are now being investigated. Actigraphy can provide an accurate measurement of sleep and wake operation by means of an accelerometer and external considerations such as light intensity can be assessed by instruments. This may be more useful for detection than commonly used subjective reports of patients and could provide novel predictors of treatment response [117].

Biomarkers for transitional use are summarized in the Figure 2 effectively.

\section{CURRENT CHALLENGES AND FUTURE PROSPECTS}

The proof supports a common pattern with each of the 


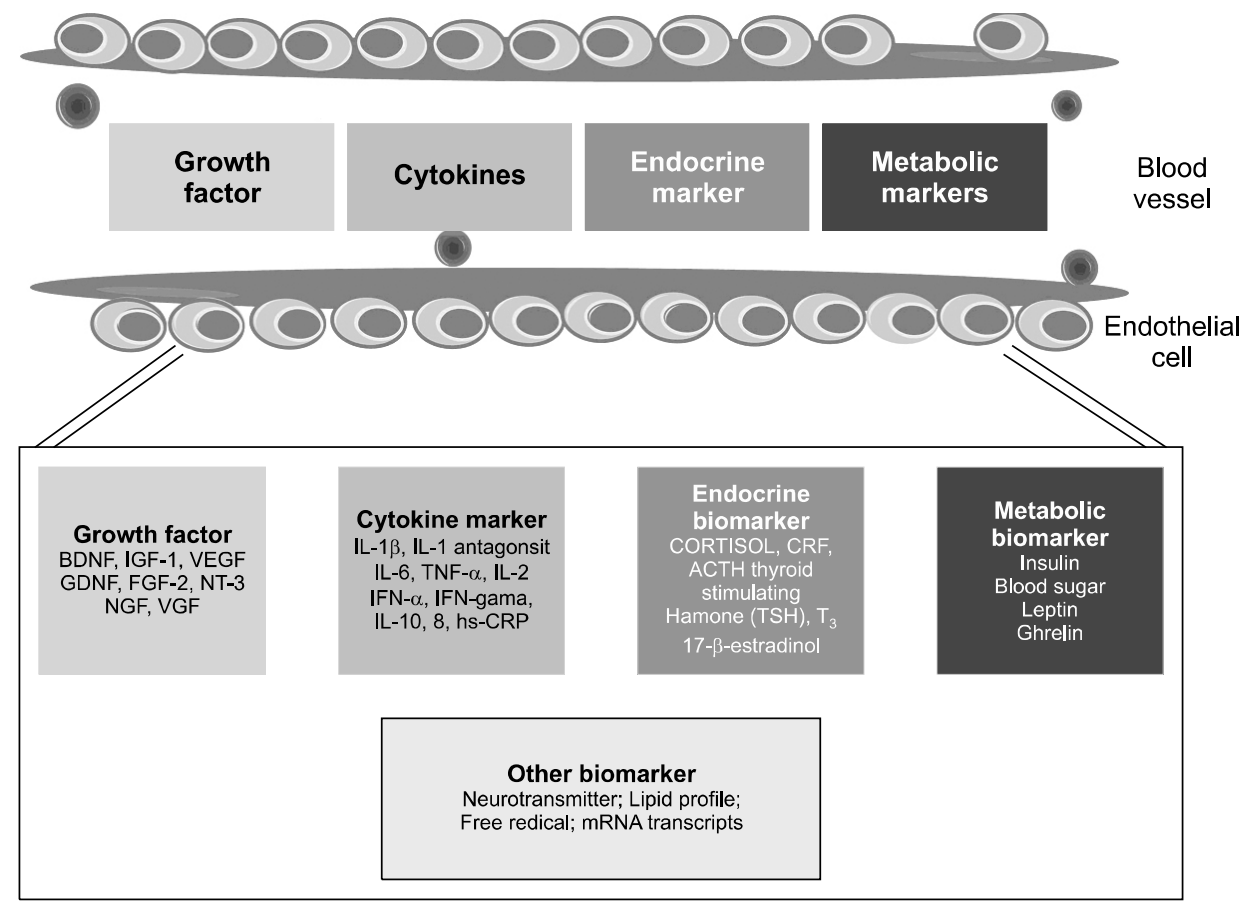

Fig. 2. Biomarker panels for MDD.

BDNF, brain-derived neurotrophic factor; IGF-1, insulin-like growth factor-1; IL, interleukin; TNF- $\alpha$, tumour necrosis factor alpha; IFN-gama, tumour necrosis fact subfamily; hs-CRP, high-sensitivity C-reactive protein; CRF, corticotrophin releasing factor; ACTH, adrenocorticotropic hormone; VEGF, vascular endothelial growth factor; FGF, fibroblast growth factor; NT-3, neurotrophin-3; NGF, nerve growth factor; MDD, major depressive disorder; GDNF, Glial cell-derived neurotrophic factor; VGF, vascular growth factor.

neuropsychological mechanisms as explained: multiple biomarkers occur and are in certain ways correlated with depression. Often such markers are intertwined in a dynamic, hard-to-model way. The proof is contradictory, and some are possibly epiphenomena of certain variables and those in a subset of patients are significant. Biomarkers may be of use in different ways (such as those which anticipate subsequent therapy outcomes, suggest that particular therapies are more likely to be successful, or which change treatments, independent of clinical improvement). New approaches to improve the uniformity and therapeutic applicability of biological tests of psychiatric communities are needed.

The research results to date continue to be repeated in large studies. Of new biomarkers, for example the chemokine thymus and activation regulated chemokines, and the tyrosine kinase 2 growth factor, that we recognize have not been tested in stable and clinically stressed test samples. Big-data studies shall test comprehensive biomarker panels for detailed study of marker associations and other improvements utilizing sumptuous analytical methods in clinical or non-clinical populations. Moreover, major component research replications may establish strongly clustered classes of biomarkers and can guide the usage of "composites" in biological psychiatry and will increase the homogeneity of potential tests.

Both widely recommended depression therapies must be carefully assessed and took into consideration the efficacy of medical trials as to their biological impact. It makes constructions surrounding biomarkers and signs to forecast effects in more customized manner in a number in therapeutic medications in conjunction with both unipolar and depressive disorder. This is potentially beneficial for both existing and established future therapies.

Nevertheless, prospective clinical studies will follow an integrative approach to biomarker work in order to meet the goals of the RDoC and concentrate on different subtypes. In order to accurately reflect the community, the experiments have to employ a significant number of people. Both exploration and guided research can be achieved for the amount of data that can potentially be obtained. The majority of resources mentioned above are well functional so that new hits for the MDD can be found. The previously involved markers, such as those de- 
scribed above, will also be replicated and explored more [118].

Biomarkers are likely to be useful through a variety of routes (e.g., those that predict subsequent response to treatment, those indicating specific treatments as more likely to be effective or those that alter with interventions regardless of clinical improvements). Novel methods are required to maximize consistency and clinical applicability of biologic assessments in psychiatric populations.

The effective implication of the above methods will contribute to improved modeling of therapeutic resistance. This will lead to more effective and sustainable care intervention interventions (e.g., long-term interventions). Assessing other relevant patient well-being metrics (e.g., quality of life and day-to-day operation) may include more thorough assessment of care results and could include biomarkers more closely. Although biological activity itself may not be able to differentiate between treatment responders and non-respondents, concurrent measurement the design of a statistical model of inadequate patient response with biomarker data may combine biomarkers with psychological or demographic parameters. In a broader sample monitored, if a credible model is built to forecast and validated retroactively the answer (either for the distressed community or a subpopulation) translation architecture may be developed. The basic biochemical results of all generally administered depressive medications were analyzed in depth, taking into consideration the validity of the therapy findings as well. It can be used to predict the results of a variety of anti-depressant treatments in more personal ways by constructs relating to biomarkers and symptoms and both unipolar and bipolar depression. This is potentially helpful for future therapies as well as for the therapies already suggested.

As a consequence, there was no individual or set of biomarkers for diagnosing depression or for guiding therapy selection. The heterogeneous depression poses a specific obstacle to the identification of biomarkers, although it offers the capacity to combine other fundamental biomarkers (i.e., a biosignature) the continued development of biomarker analysis and analytical instruments are needed, as these are reliable techniques. These are essential for tailoring depression therapies in particular individuals, these technical advances along with the through detection of possible biomarkers will potentially contribute to faster and successful diagnosis. It would improve.

\section{CONCLUSION}

By this review we summarize all the diverse biomarkers assuming a significant job in the location or treatment of the distinctive MDD. The vast research on depression indicates a large variety of biomarkers that may help patients with depression enhance their diagnosis. The use of the above biomarkers will likely lead to improved ability to prospectively estimate treatment resistance. This can be supported by more authentic and persistent treatment response measures (e.g., long term). The evaluation of other valid patient wellness measures (such as quality of life and day-to-day functioning) could provide a holistic assessment of the results of treatment that could involve biomarkers more closely. While biological activity alone cannot differentiate between patient respondents and non-respondents, in establishing a predictive model for inadequate treatment response, concurrent measurements of biomarkers with psychosocial or demographic variables may be paired with biomarker knowledge. When a reliable model is developed for predicting responses (both in the low population and subpopulation) and retrospectively validated, a translational design can be used in a large controlled trial. In addition to neurotransmitters and neuroendocrine receptors, the inflammatory reaction (and the immune system in general), Metabolist and growth factors that have been subject to extensiveness for decades have been illustrated in recent studies. Excessive opposing data however suggests that a range of issues need to be addressed in order to enhance the diagnosis and treatment of people with depression before biomarker testing can be implemented. Such markers can display the greatest potential in a subgroup of patients to predict care reaction, and combined assessment of biological and psychological data can increase the capacity of those at risk of weak therapy to recognise prospectively. The review additionally gives a review of various biomarker's assuming a potential job in neurodegenerative illness.

\section{Acknowledgments}

The authors gratefully acknowledge Dr. Madhu Chitkara, Vice Chancellor, Chitkara University, Rajpura, Punjab, India, and Dr. Sandeep Arora, Director, Chitkara College of Pharmacy, Chitkara University, Rajpura, Punjab, India for support and institutional facilities. 
Conflicts of Interest

No potential conflict of interest relevant to this article was reported.

\section{Author Contributions}

Conceptualization: Ravinder Singh. Design: Sahil Malik, Govind Arora. Supervision: Ravinder Singh. Resources: Ravinder Singh. Materials: Govind Arora, Sahil Malik. Data collection and processing: Govind Arora, Sahil Malik. Analysis and interpretation: Ravinder Singh, Govind Arora, Sahil Malik. Literature search: Govind Arora, Akriti Dangol, Sanjay Goyal, Sahil Malik. Writing manuscript: Sahil Malik, Govind Arora. Critical review: Ravinder Singh, Govind Arora, Sahil Malik.

\section{ORCID}

Sahil Malik

Ravinder Singh

https://orcid.org/0000-0002-8156-7601

Govind Arora https://orcid.org/0000-0003-1565-9740

Akriti Dangol https://orcid.org/0000-0001-9481-6868

Sanjay Goyal https://orcid.org/0000-0002-5495-1034 https://orcid.org/0000-0002-7867-1280

\section{REFERENCES}

1. Strawbridge R, Young AH, Cleare AJ. Biomarkers for depression: recent insights, current challenges and future prospects. Focus 2018;16:194-209.

2. Aydemir O, Deveci A, Taneli F. The effect of chronic antidepressant treatment on serum brain-derived neurotrophic factor levels in depressed patients: a preliminary study. Prog Neuropsychopharmacol Biol Psychiatry 2005;29:261-265.

3. Mercier A, Auger-Aubin I, Lebeau JP, Schuers M, Boulet P, Hermil JL, et al. Evidence of prescription of antidepressants for non-psychiatric conditions in primary care: an analysis of guidelines and systematic reviews. BMC Fam Pract 2013;14:55.

4. Freland L, Beaulieu JM. Inhibition of GSK3 by lithium, from single molecules to signaling networks. Front Mol Neurosci 2012;5:14.

5. Begić D, Mahnik-Milos M, Grubisin J. EEG characteristics in depression, "negative" and "positive" schizophrena. Psychiatr Danub 2009;21:579-584.

6. de la Torre C, Ceña V. The delivery challenge in neurodegenerative disorders: the nanoparticles role in Alzheimer's disease therapeutics and diagnostics. Pharmaceutics 2018; 10:190.

7. Bienvenu OJ, Davydow DS, Kendler KS. Psychiatric 'diseases' versus behavioral disorders and degree of genetic influence. Psychol Med 2011;41:33-40.
8. Li M, Soczynska JK, Kennedy SH. Inflammatory biomarkers in depression: an opportunity for novel therapeutic interventions. Curr Psychiatry Rep 2011;13:316-320.

9. Nestler EJ. Epigenetic mechanisms of depression. JAMA Psychiatry 2014;71:454-456.

10. Lakhan SE, Vieira K, Hamlat E. Biomarkers in psychiatry: drawbacks and potential for misuse. Int Arch Med 2010;3:1.

11. Krishnan V, Nestler EJ. The molecular neurobiology of depression. Nature 2008;455:894-902.

12. Alexander RC, Preskorn S. Clinical pharmacology in the development of new antidepressants: the challenges. Curr Opin Pharmacol 2014;14:6-10.

13. Warden D, Rush AJ, Trivedi MH, Fava M, Wisniewski SR. The STAR*D Project results: a comprehensive review of findings. Curr Psychiatry Rep 2007;9:449-459.

14. Anderson HD, Pace WD, Libby AM, West DR, Valuck RJ. Rates of 5 common antidepressant side effects among new adult and adolescent cases of depression: a retrospective US claims study. Clin Ther 2012;34:113-123.

15. Sawada N, Uchida H, Suzuki T, Watanabe K, Kikuchi T, Handa T, et al. Persistence and compliance to antidepressant treatment in patients with depression: a chart review. BMC Psychiatry 2009;9:38.

16. Monteggia LM, Zarate C Jr. Antidepressant actions of ketamine: from molecular mechanisms to clinical practice. Curr Opin Neurobiol 2015;30:139-143.

17. Zarate CA, Manji HK. Riluzole in psychiatry: a systematic review of the literature. Expert Opin Drug Metab Toxicol 2008;4:1223-1234.

18. Casacalenda N, Perry JC, Looper K. Remission in major depressive disorder: a comparison of pharmacotherapy, psychotherapy, and control conditions. Am I Psychiatry 2002; 159:1354-1360.

19. Carpenter LL, Janicak PG, Aaronson ST, Boyadjis T, Brock DG, Cook IA, et al. Transcranial magnetic stimulation (TMS) for major depression: a multisite, naturalistic, observational study of acute treatment outcomes in clinical practice. Depress Anxiety 2012;29:587-596.

20. Holtzheimer PE, Mayberg HS. Deep brain stimulation for psychiatric disorders. Annu Rev Neurosci 2011;34:289-307.

21. Brewer HB Jr, Fairwell T, Kay L, Meng M, Ronan R, Law S, et al. Human plasma proapoA-l: isolation and amino-terminal sequence. Biochem Biophys Res Commun 1983;113:626-632.

22. Suravajhala P, Kogelman LJ, Kadarmideen HN. Multi-omic data integration and analysis using systems genomics approaches: methods and applications in animal production, health and welfare. Genet Sel Evol 2016;48:38.

23. Menke A. Gene expression: biomarker of antidepressant therapy? Int Rev Psychiatry 2013;25:579-591.

24. Peng B, Li H, Peng XX. Functional metabolomics: from biomarker discovery to metabolome reprogramming. Protein Cell 2015;6:628-637.

25. Aagaard K, Petrosino J, Keitel W, Watson M, Katancik J, 
Garcia N, et al. The Human Microbiome Project strategy for comprehensive sampling of the human microbiome and why it matters. FASEB J 2013;27:1012-1022.

26. Sonner Z, Wilder E, Heikenfeld J, Kasting G, Beyette F, Swaile D, et al. The microfluidics of the eccrine sweat gland, including biomarker partitioning, transport, and biosensing implications. Biomicrofluidics 2015;9:031301.

27. Hendrickx H, McEwen BS, Ouderaa Fv. Metabolism, mood and cognition in aging: the importance of lifestyle and dietary intervention. Neurobiol Aging 2005;26 Suppl 1:1-5.

28. Tseng PT, Cheng YS, Chen YW, Wu CK, Lin PY. Increased levels of vascular endothelial growth factor in patients with major depressive disorder: a meta-analysis. Eur Neuropsychopharmacol 2015;25:1622-1630.

29. Kaufman J, DeLorenzo C, Choudhury S, Parsey RV. The 5-HT1A receptor in major depressive disorder. Eur Neuropsychopharmacol 2016;26:397-410.

30. Coplan JD, Gopinath S, Abdallah CG, Berry BR. A neurobiological hypothesis of treatment-resistant depression mechanisms for selective serotonin reuptake inhibitor non-efficacy. Front Behav Neurosci 2014;8:189.

31. Hasler G, Drevets WC, Manji HK, Charney DS. Discovering endophenotypes for major depression. Neuropsychopharmacology 2004;29:1765-1781.

32. Hiles SA, Attia J, Baker AL. Changes in interleukin-6, C-reactive protein and interleukin-10 in people with depression following antidepressant treatment: a meta-analysis. Brain Behav Immun 2010;24 Suppl 1:S44.

33. Molendijk ML, Spinhoven P, Polak M, Bus BA, Penninx BW, Elzinga BM. Serum BDNF concentrations as peripheral manifestations of depression: evidence from a systematic review and meta-analyses on 179 associations ( $\mathrm{N}=9484)$. Mol Psychiatry 2014;19:791-800.

34. Lin E, Chen PS. Pharmacogenomics with antidepressants in the STAR*D study. Pharmacogenomics 2008;9:935-946.

35. Strawbridge R, Arnone D, Danese A, Papadopoulos A, Herane Vives A, Cleare AJ. Inflammation and clinical response to treatment in depression: a meta-analysis. Eur Neuropsychopharmacol 2015;25:1532-1543.

36. Horowitz MA, Zunszain PA. Neuroimmune and neuroendocrine abnormalities in depression: two sides of the same coin. Ann N Y Acad Sci 2015;1351:68-79.

37. Juruena MF, Cleare AJ. [Overlap between atypical depression, seasonal affective disorder and chronic fatigue syndrome]. Braz J Psychiatry 2007;29 Suppl 1:S19-S26. Portuguese.

38. Beauchaine TP. The role of biomarkers and endophenotypes in prevention and treatment of psychopathological disorders. Biomark Med 2009;3:1-3.

39. LaBaer J. So, you want to look for biomarkers (introduction to the special biomarkers issue). J Proteome Res 2005;4: 1053-1059.

40. Pepe MS, Janes H. Insights into latent class analysis of diagnostic test performance. Biostatistics 2007;8:474-484.
41. Le-Niculescu H, Kurian SM, Yehyawi N, Dike C, Patel SD, Edenberg HJ, et al. Identifying blood biomarkers for mood disorders using convergent functional genomics. Mol Psychiatry 2009; 14:156-174.

42. Redei EE, Andrus BM, Kwasny MJ, Seok J, Cai X, Ho J, et al. Blood transcriptomic biomarkers in adult primary care patients with major depressive disorder undergoing cognitive behavioral therapy. Transl Psychiatry 2014;4:e442.

43. Kambeitz JP, Howes OD. The serotonin transporter in depression: meta-analysis of in vivo and post mortem findings and implications for understanding and treating depression. J Affect Disord 2015;186:358-366.

44. Joensuu $M$, Ahola $P$, Knekt $P$, Lindfors $O$, Saarinen $P$, Tolmunen $\mathrm{T}$, et al. Baseline symptom severity predicts serotonin transporter change during psychotherapy in patients with major depression. Psychiatry Clin Neurosci 2016;70: 34-41.

45. White KJ, Walline CC, Barker EL. Serotonin transporters: implications for antidepressant drug development. In: Rapaka $R S$, Sadée W, editors. Drug addiction. New York:Springer; 2008. p. 193-215.

46. Björk K, Sjögren B, Svenningsson P. Regulation of serotonin receptor function in the nervous system by lipid rafts and adaptor proteins. Exp Cell Res 2010;316:1351-1356.

47. Rivera-Baltanas T, Olivares JM, Calado-Otero M, Kalynchuk LE, Martinez-Villamarin JR, Caruncho HJ. Serotonin transporter clustering in blood lymphocytes as a putative biomarker of therapeutic efficacy in major depressive disorder. J Affect Disord 2012;137:46-55.

48. Ersche KD, Roiser JP, Lucas M, Domenici E, Robbins TW, Bullmore ET. Peripheral biomarkers of cognitive response to dopamine receptor agonist treatment. Psychopharmacology (Berl) 2011;214:779-789.

49. López León S, Croes EA, Sayed-Tabatabaei FA, Claes S, Van Broeckhoven C, van Duijn CM. The dopamine D4 receptor gene 48-base-pair-repeat polymorphism and mood disorders: a meta-analysis. Biol Psychiatry 2005;57:999-1003.

50. Burnashev N, Szepetowski P. NMDA receptor subunit mutations in neurodevelopmental disorders. Curr Opin Pharmacol 2015;20:73-82.

51. Gray AL, Hyde TM, Deep-Soboslay A, Kleinman JE, Sodhi MS. Sex differences in g/utamate receptor gene expression in major depression and suicide. Mol Psychiatry 2015;20: 1057-1068.

52. Petty F, Kramer GL, Fulton M, Davis L, Rush AJ. Stability of plasma GABA at four-year follow-up in patients with primary unipolar depression. Biol Psychiatry 1995;37:806-810.

53. Küçükibrahimoğlu E, Saygin MZ, Calişkan M, Kaplan OK, Unsal C, Gören MZ. The change in plasma GABA, glutamine and glutamate levels in fluoxetine- or S-citalopramtreated female patients with major depression. Eur J Clin Pharmacol 2009;65:571-577.

54. Devanand DP, Shapira B, Petty F, Kramer G, Fitzsimons L, 
Lerer B, et al. Effects of electroconvulsive therapy on plasma GABA. Convuls Ther 1995;11:3-13.

55. Pehrson AL, Sanchez C. Altered $\gamma$-aminobutyric acid neurotransmission in major depressive disorder: a critical review of the supporting evidence and the influence of serotonergic antidepressants. Drug Des Devel Ther 2015;9:603-624.

56. Bak LK, Schousboe A, Waagepetersen HS. The g/utamate/ GABA-g/utamine cycle: aspects of transport, neurotransmitter homeostasis and ammonia transfer. I Neurochem 2006;98:641-653.

57. Maddock RJ, Buonocore MH. MR spectroscopic studies of the brain in psychiatric disorders. In: Carter C, Dalley J, editors. Brain imaging in behavioral neuroscience. Berlin, Heidelberg:Springer;2011. p.199-251.

58. Dager SR, Corrigan NM, Richards TL, Posse S. Research applications of magnetic resonance spectroscopy to investigate psychiatric disorders. Top Magn Reson Imaging 2008; 19:81-86.

59. Feyissa AM, Chandran A, Stockmeier CA, Karolewicz B. Reduced levels of $N R 2 A$ and $N R 2 B$ subunits of $N M D A$ receptor and PSD-95 in the prefrontal cortex in major depression. Prog Neuropsychopharmacol Biol Psychiatry 2009;33:70-75.

60. Dos Santos MCT, Barreto-Sanz MA, Correia BRS, Bell R, Widnall C, Perez LT, et al. miRNA-based signatures in cerebrospinal fluid as potential diagnostic tools for early stage Parkinson's disease. Oncotarget 2018;9:17455-17465.

61. Duman RS. Neurobiology of stress, depression, and rapid acting antidepressants: remodeling synaptic connections. Depress Anxiety 2014;31:291-296.

62. Dunlop BW, Mayberg HS. Neuroimaging-based biomarkers for treatment selection in major depressive disorder. Dialogues Clin Neurosci 2014;16:479-490.

63. Emamzadeh FN. Alpha-synuclein structure, functions, and interactions. J Res Med Sci 2016;21:29.

64. Felger JC, Lotrich FE. Inflammatory cytokines in depression: neurobiological mechanisms and therapeutic implications. Neuroscience 2013;246:199-229.

65. Fernandes BS, Gama CS, Kauer-Sant'Anna M, Lobato MI, Belmonte-de-Abreu P, Kapczinski F. Serum brain-derived neurotrophic factor in bipolar and unipolar depression: a potential adjunctive tool for differential diagnosis. J Psychiatr Res 2009:43:1200-1204.

66. Castrén E, Rantamäki T. The role of BDNF and its receptors in depression and antidepressant drug action: reactivation of developmental plasticity. Dev Neurobiol 2010;70:289-297.

67. Clark-Raymond A, Halaris A. VEGF and depression: a comprehensive assessment of clinical data. J Psychiatr Res 2013; 47:1080-1087.

68. Malberg JE, Monteggia LM. VGF, a new player in antidepressant action? Sci Signal 2008;1:pe19.

69. Turner CA, Akil H, Watson SJ, Evans SJ. The fibroblast growth factor system and mood disorders. Biol Psychiatry
2006;59:1128-1135.

70. Bernard R, Kerman IA, Thompson RC, Jones EG, Bunney WE, Barchas JD, et al. Altered expression of glutamate signaling, growth factor, and glia genes in the locus coeruleus of patients with major depression. Mol Psychiatry 2011;16:634646.

71. Evans SJ, Choudary PV, Neal CR, Li JZ, Vawter MP, Tomita $\mathrm{H}$, et al. Dysregulation of the fibroblast growth factor system in major depression. Proc Natl Acad Sci U S A 2004;101: 15506-15511.

72. Gaughran F, Payne J, Sedgwick PM, Cotter D, Berry M. Hippocampal FGF-2 and FGFR1 mRNA expression in major depression, schizophrenia and bipolar disorder. Brain Res Bull 2006;70:221-227.

73. Smith RS. The macrophage theory of depression. Med Hypotheses 1991;35:298-306.

74. Irwin MR, Miller AH. Depressive disorders and immunity: 20 years of progress and discovery. Brain Behav Immun 2007;21:374-383.

75. Maes M, Leonard BE, Myint AM, Kubera M, Verkerk R. The new '5-HT' hypothesis of depression: cell-mediated immune activation induces indoleamine 2,3-dioxygenase, which leads to lower plasma tryptophan and an increased synthesis of detrimental tryptophan catabolites (TRYCATs), both of which contribute to the onset of depression. Prog Neuropsychopharmacol Biol Psychiatry 2011;35:702-721.

76. Miller $\mathrm{AH}$, Maletic $\mathrm{V}$, Raison CL. Inflammation and its discontents: the role of cytokines in the pathophysiology of major depression. Biol Psychiatry 2009;65:732-741.

77. Miller AH, Raison CL. The role of inflammation in depression: from evolutionary imperative to modern treatment target. Nat Rev Immunol 2016;16:22-34.

78. Raison CL, Capuron L, Miller AH. Cytokines sing the blues: inflammation and the pathogenesis of depression. Trends Immunol 2006;27:24-31.

79. Eyre HA, Air T, Pradhan A, Johnston J, Lavretsky H, Stuart MJ, et al. A meta-analysis of chemokines in major depression. Prog Neuropsychopharmacol Biol Psychiatry 2016;68:1-8.

80. Raison CL, Felger JC, Miller AH. Inflammation and treatment resistance in major depression: the perfect storm. Psychiatr Times 2013;30.

81. Haapakoski R, Mathieu J, Ebmeier KP, Alenius H, Kivimäki M. Cumulative meta-analysis of interleukins 6 and $1 \beta$, tumour necrosis factor $\alpha$ and $C$-reactive protein in patients with major depressive disorder. Brain Behav Immun 2015; 49:206-215.

82. Liu Y, Ho RC, Mak A. Interleukin (IL)-6, tumour necrosis factor alpha (TNF- $\alpha$ ) and soluble interleukin-2 receptors (s/L-2R) are elevated in patients with major depressive disorder: a meta-analysis and meta-regression. I Affect Disord 2012; 139:230-239.

83. Farooq RK, Asghar K, Kanwal S, Zulqernain A. Role of inflammatory cytokines in depression: focus on interleukin-1 $\beta$. 
Biomed Rep 2017;6:15-20.

84. Cattaneo A, Ferrari C, Uher R, Bocchio-Chiavetto L, Riva MA, MRC ImmunoPsychiatry Consortium, et al. Absolute measurements of macrophage migration inhibitory factor and interleukin-1- $\beta$ mRNA levels accurately predict treatment response in depressed patients. Int I Neuropsychopharmacol 2016;19:pyw045.

85. Baune BT, Smith E, Reppermund S, Air T, Samaras K, Lux O, et al. Inflammatory biomarkers predict depressive, but not anxiety symptoms during aging: the prospective Sydney Memory and Aging Study. Psychoneuroendocrinology 2012;37:1521-1530.

86. Fornaro M, Rocchi G, Escelsior A, Contini P, Martino M. Might different cytokine trends in depressed patients receiving duloxetine indicate differential biological backgrounds. J Affect Disord 2013;145:300-307.

87. Hernández ME, Mendieta D, Martínez-Fong D, Loría F, Moreno J, Estrada I, et al. Variations in circulating cytokine levels during 52 week course of treatment with SSRI for major depressive disorder. Eur Neuropsychopharmacol 2008; 18:917-924.

88. Hannestad J, DellaGioia N, Bloch M. The effect of antidepressant medication treatment on serum levels of inflammatory cytokines: a meta-analysis. Neuropsychopharmacology 2011;36:2452-2459.

89. Srikanthan K, Feyh A, Visweshwar H, Shapiro JI, Sodhi K. Systematic review of metabolic syndrome biomarkers: a panel for early detection, management, and risk stratification in the West Virginian population. Int J Med Sci 2016; 13:25-38.

90. Lu XY. The leptin hypothesis of depression: a potential link between mood disorders and obesity? Curr Opin Pharmacol 2007;7:648-652.

91. Wittekind DA, Kluge M. Ghrelin in psychiatric disorders - a review. Psychoneuroendocrinology 2015;52:176-194.

92. Kan C, Silva N, Golden SH, Rajala U, Timonen M, Stahl D, et al. A systematic review and meta-analysis of the association between depression and insulin resistance. Diabetes Care 2013;36:480-489.

93. Liu X, Li J, Zheng P, Zhao X, Zhou C, Hu C, et al. Plasma lipidomics reveals potential lipid markers of major depressive disorder. Anal Bioanal Chem 2016;408:6497-6507.

94. Lustman PJ, Anderson RJ, Freedland KE, de Groot M, Carney RM, Clouse RE. Depression and poor glycemic control: a meta-analytic review of the literature. Diabetes Care 2000; 23:934-942.

95. Maes M. Evidence for an immune response in major depression: a review and hypothesis. Prog Neuropsychopharmacol Biol Psychiatry 1995;19:11-38.

96. Pan A, Keum N, Okereke OI, Sun Q, Kivimaki M, Rubin RR, et al. Bidirectional association between depression and metabolic syndrome: a systematic review and meta-analysis of epidemiological studies. Diabetes Care 2012;35:1171-1180.
97. Carvalho AF, Rocha DQ, McIntyre RS, Mesquita LM, Köhler CA, Hyphantis TN, et al. Adipokines as emerging depression biomarkers: a systematic review and meta-analysis. J Psychiatr Res 2014;59:28-37.

98. Lamers F, Vogelzangs N, Merikangas KR, de Jonge P, Beekman AT, Penninx BW. Evidence for a differential role of HPA-axis function, inflammation and metabolic syndrome in melancholic versus atypical depression. Mol Psychiatry 2013;18: 692-699.

99. Zheng $\mathrm{H}$, Zheng $\mathrm{P}$, Zhao L, Jia J, Tang S, Xu P, et al. Predictive diagnosis of major depression using NMR-based metabolomics and least-squares support vector machine. Clin Chim Acta 2017;464:223-227.

100. Liu T, Zhong S, Liao X, Chen J, He T, Lai S, et al. A meta-analysis of oxidative stress markers in depression. PloS one 2015; 10(10):e0138904.

101. Foulds PG, Mitchell JD, Parker A, Turner R, Green G, Diggle $\mathrm{P}$, et al. Phosphorylated $\alpha$-synuclein can be detected in blood plasma and is potentially a useful biomarker for Parkinson's disease. FASEB J 2011;25:4127-4137.

102. Fronczek R, Overeem S, Lee SY, Hegeman IM, van Pelt J, van Duinen SG, et al. Hypocretin (orexin) loss in Parkinson's disease. Brain 2007;130(Pt 6):1577-1585.

103. Maes M, Mihaylova I, Kubera M, Uytterhoeven M, Vrydags $\mathrm{N}$, Bosmans E. Increased 8-hydroxy-deoxyguanosine, a marker of oxidative damage to DNA, in major depression and myalgic encephalomyelitis / chronic fatigue syndrome. Neuro Endocrinol Lett 2009;30:715-722.

104. Forlenza MJ, Miller GE. Increased serum levels of 8-hydroxy-2'-deoxyguanosine in clinical depression. Psychosom Med 2006;68:1-7.

105. Jorgensen A, Krogh J, Miskowiak K, Bolwig TG, Kessing LV, Fink-Jensen $\mathrm{A}$, et al. Systemic oxidatively generated DNA/RNA damage in clinical depression: associations to symptom severity and response to electroconvulsive therapy. J Affect Disord 2013;149:355-362.

106. lida $\mathrm{T}$, Chikamura $\mathrm{C}$, Inoue $\mathrm{K}$, Ito $\mathrm{Y}$, Ishikawa $\mathrm{H}$, Teradaira $\mathrm{R}$, et al. Association of STAl and SDS scores with 8-hydroxydeoxyguanosine and serotonin levels in young women with depressive symptoms. J Neuropsychiatry Clin Neurosci 2011;23:E10.

107. Yi S, Nanri A, Matsushita $\mathrm{Y}$, Kasai $\mathrm{H}$, Kawai K, Mizoue T. Depressive symptoms and oxidative DNA damage in Japanese municipal employees. Psychiatry Res 2012;200: 318-322.

108. Chung CP, Schmidt D, Stein CM, Morrow JD, Salomon RM. Increased oxidative stress in patients with depression and its relationship to treatment. Psychiatry Res 2013;206:213-216.

109. Gururajan A, Clarke G, Dinan TG, Cryan JF. Molecular biomarkers of depression. Neurosci Biobehav Rev 2016;64: 101-133.

110. Parker KJ, Schatzberg AF, Lyons DM. Neuroendocrine aspects of hypercortisolism in major depression. Horm Behav 
2003;43:60-66.

111. Stetler C, Miller GE. Depression and hypothalamic-pituitary-adrenal activation: a quantitative summary of four decades of research. Psychosom Med 2011;73:114-126.

112. Herane Vives A, De Angel V, Papadopoulos A, Strawbridge $\mathrm{R}$, Wise $\mathrm{T}$, Young $\mathrm{AH}$, et al. The relationship between cortisol, stress and psychiatric illness: New insights using hair analysis. J Psychiatr Res 2015;70:38-49.

113. Fischer S, Strawbridge R, Vives AH, Cleare AJ. Cortisol as a predictor of psychological therapy response in depressive disorders: systematic review and meta-analysis. Br J Psychiatry 2017;210:105-109.

114. Anacker C, Zunszain PA, Carvalho LA, Pariante CM. The glucocorticoid receptor: pivot of depression and of antidepressant treatment? Psychoneuroendocrinology 2011;36:
415-425.

115. Markopoulou K, Papadopoulos A, Juruena MF, Poon L, Pariante CM, Cleare AJ. The ratio of cortisol/DHEA in treatment resistant depression. Psychoneuroendocrinology 2009;34:19-26.

116. Marsden WN. Synaptic plasticity in depression: molecular, cellular and functional correlates. Prog Neuropsychopharmacol Biol Psychiatry 2013;43:168-184.

117. Duman RS, Voleti B. Signaling pathways underlying the pathophysiology and treatment of depression: novel mechanisms for rapid-acting agents. Trends Neurosci 2012;35:47-56.

118. Trivedi MH, McGrath PJ, Fava M, Parsey RV, Kurian BT, Phillips ML, et al. Establishing moderators and biosignatures of antidepressant response in clinical care (EMBARC): rationale and design. J Psychiatr Res 2016;78:11-23. 